Original Research

\title{
The Relationship between Sensory Reactivity Differences and Mental Health Symptoms in Children with Neurodevelopmental Conditions and Their Neurotypical Peers
}

Timothy Rossow ${ }^{1,+, *}$, Elysa J Marco ${ }^{2,3,{ }^{+}, \text {Molly Gerdes }}{ }^{2,+}$, Teresa Tavassoli ${ }^{1,{ }^{+}}$

1. School of Psychology and Clinical Language Sciences, University of Reading, UK; E-Mails: t.w.I.rossow@pgr.reading.ac.uk; t.tavassoli@reading.ac.uk

2. Cortica, San Rafael, California, USA; E-Mails: emarco@corticacare.com; mgerdes@corticacare.com

3. Department of Radiology, University of California San Francisco, California, USA

$\dagger$ These authors contributed equally to this work.

* Correspondence: Timothy Rossow; E-Mail: t.w.I.rossow@pgr.reading.ac.uk

Academic Editor: Bart Ellenbroek

Special Issue: Sensory reactivity in neurodevelopmental conditions

OBM Neurobiology

2021, volume 5 , issue 4

doi:10.21926/obm.neurobiol.2104110
Received: July 28, 2021

Accepted: October 14, 2021

Published: October 25, 2021

\begin{abstract}
Children with neurodevelopmental conditions (NDC) often have sensory reactivity differences, such as being overwhelmed by sounds, which can elicit distress in their everyday life. Furthermore, children with an NDC often present with a greater frequency of mental health symptoms compared to their typically developing peers. However little research has examined the relationship of these sensory reactivity differences on broader mental health. This study set out to explore the relationship between sensory reactivity and mental health symptoms. Data from parent-reported measures of sensory reactivity and mental health symptoms were collected for 49 children presenting with a neurodevelopmental condition and 42 typically developing (TD) controls. Partial correlational analyses, controlling for IQ, were used to elucidate the relationship between sensory reactivity and mental health
\end{abstract}

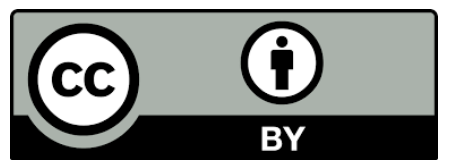

(C) 2021 by the author. This is an open access article distributed under the conditions of the Creative Commons by Attribution License, which permits unrestricted use, distribution, and reproduction in any medium or format, provided the original work is correctly cited. 
symptoms. Children with an NDC were significantly more likely to present with a sensory reactivity difference or mental health symptom. Sensory seeking was found to be the sensory subtype most related to mental health symptoms, especially in externalising conditions. There was evidence also found for a relationship between depression, sensory seeking and sensory hypo-reactivity, and GAD and hyper-reactivity. Findings suggest a relationship between sensory reactivity and mental health symptoms in children with a neurodevelopmental condition. There is also preliminary evidence of unique sensory-based profiles of mental health. Assessment of sensory difficulties as part of more rigorous mental health assessments within mental health services will support more accurate diagnoses and intervention.

\section{Keywords}

Sensory reactivity; neurodevelopmental conditions; mental health; sensory

\section{Introduction}

Sensory reactivity symptoms, defined as different responses to sensory stimuli in the environment [1], are often present in neurodevelopmental conditions (NDC), including in those with categorical labels such as autism [2-4]. These sensory reactivity differences have been shown to present across the visual, auditory, tactile, vestibular, proprioceptive, gustatory and olfactory modalities, with some modalities affected more than others $[5,6]$. Further, research has identified three broad sensory modulation patterns: hyper-reactivity (aka sensitivity to sensory input; overresponsivity), hypo-reactivity (aka poor registration of sensory input; under-responsivity) and sensory seeking (aka sensory craving) [5, 7]. Additionally, individuals with an NDC are at a significantly higher risk of developing or being diagnosed with a comorbid psychiatric condition than their typically developing peers, with prevalence rates of comorbid mental health diagnoses in NDC ranging from $25 \%$ to $95 \%$ [8-14]. Until now, NDC research exploring comorbid mental health has primarily investigated the increased prevalence through social-psychological (e.g. societal stigma and its negative impact on self-esteem) or biological mechanisms (e.g. amygdala volume, genetics) [15-18]. However, little research has explored the impact of sensory reactivity symptoms and their relationship with mental health symptoms. The research available has focused on individual sensory reactivity constructs within specific diagnoses, particularly hyper-reactivity and anxiety [19-21]. A recent review of the National Institute of Mental Health's (NIMH) Research Domain Criteria (RDoC) framework has further highlighted the lack of research into sensory reactivity and mental health as symptoms across conditions, and identified the need for further investigation across mental health diagnoses in NDC [22]. It is this gap which the current study targets. Herein, we seek to address the correlational relationship between sensory reactivity and mental health constructs across children with an NDC and typically developing children without sensory symptoms. 


\section{Materials and Methods}

\subsection{Participants}

In total 91 children took part, of which 49 children had a neurodevelopmental diagnosis (26 children had an autism spectrum condition (ASC) [7 - 11 years, $M=9.46, S D=1.33$ ], 23 children had sensory processing disorder (SPD) $[7-12$ years, $M=9.15, S D=1.24]$ ) and 42 typically developing children ( $7-12$ years, $M=9.74, S D=1.52)$. Participants were from the University of California San Francisco's (UCSF) Sensory Neurodevelopment and Autism Program (SNAP) registry and database. Written informed consent was obtained from parents for all participants, and the children provided informed assent which was witnessed and formally recorded. Exclusion criteria for all participants were brain malformation or injury, movement disorder, hearing impairment, or Perceptual Reasoning Index (PRI) score $<70$ on the Wechsler Intelligence Scale for Children-Fourth Edition. Inclusion criteria for autistic ${ }^{1}$ participants were a community diagnosis of ASC, a score of $\geq 15$ on the Social Communication Questionnaire (SCQ) [24] and a confirmed ASC classification with the Autism Diagnostic Observation Schedule, Second Edition (ADOS-2) [25]. Autistic participants had a mean full-scale IQ score of 97.73 (SD = 12.30) obtained from the Wechsler Intelligence Scale for Children, Fourth Editions (WISC-IV) [25]. All autistic participants completed the ADOS-2 Module 3, with severity scores ranging from 3 to $10(M=6.41$; SD = 1.82). Inclusion criteria for the SPD participants included a standing diagnosis of SPD from a community occupational therapist, as well as a score on the Sensory Profile [27] in the "Definite Difference" range ( $<2 \%$ probability) in one or more of the sensory domains (auditory, visual, oral/olfactory, tactile, vestibular, or multisensory). SPD participants had a mean full-scale IQ score of 108.77 (SD = 15.66) obtained from the WISC-IV. Inclusion criteria for TD participants included no standing diagnoses and who were not awaiting or flagged for diagnostic assessment. TD participants had a mean full-scale IQ score of 116.80 (SD = 12.27) obtained from the WISC-IV. Three autistic participants and 21 SPD participants had a comorbid diagnosis of ADHD. All autistic participants were male $(n=26)$, whereas there was a mix of males and females in SPD participants (males $=41$, females $=14$ ) and in the typically developing group (males $=39$, females $=24$ ). Whilst participants were asked to provide information on their ethnic background, this question was not compulsory and as such many participants chose not to report their ethnicity. Participants who did provide this data reported Caucasian (86.7\%), Asian (6.7\%) and mixed (6.7\%) backgrounds.

The authors assert that all procedures contributing to this work comply with the ethical standards of the relevant national and institutional committees on human experimentation and with the Helsinki Declaration of 1975 , as revised in 2008. All procedures involving human subjects were approved by the Institutional Review Board of the University of California San Francisco (10-01940).

\footnotetext{
${ }^{1}$ Careful consideration is given to the language used within the manuscript, and autism first language has been incorporated in line with research findings [23].
} 


\subsection{Measures}

\subsubsection{The Sensory Profile - Parent (SP)}

The parent version of the Sensory Profile is a 125-question standardised measure of sensory processing [26]. Reversed scored, such that the lower the score the greater the sensory reactivity, the profile requires parents to list the frequency of their child's reactions to various common sensory stimuli, and provides domain raw scores for auditory, visual, vestibular, touch, multisensory and oral processing, and combined construct scores for sensory seeking, poor registration (hyporeactivity) and sensory avoidant (hyper-reactivity) [26]. The SP is the most commonly used sensory measure for autistic individuals [28]. The Sensory Profile has been found to have good test-retest reliability (intraclass correlation coefficients $=0.80-0.90)$, and good internal consistency ( $\alpha$ $=.89-.95)[29]$.

\subsubsection{Child Symptom Inventory Fourth Edition (CSI-4)}

The Child Symptom Inventory Fourth Edition is a parent-completed rating scale that allows for the screening of a range of emotional and behavioural conditions in children aged 5 to 12 years [30]. The CSI-4 items symbolize DSM-IV symptoms, providing symptom count scores for inattention, hyperactivity, Attention Deficit Hyperactivity Disorder (ADHD; which is a composite of inattention and hyperactivity), Oppositional Defiant Disorder (ODD), Conduct Disorder (CD), Generalized Anxiety Disorder (GAD), obsessions, compulsions, Post-Traumatic Stress Disorder (PTSD), motor and vocal tics, depression, and social phobia. Test reliability was reported to be moderate to high ( $\alpha$ $=.52-.78$, and internal consistency also moderate to high ( $\alpha=.69-.92)$ [30]. The CSI-4 has been used extensively in research with neurodiverse children (as outlined in [31] Gadow, 2019).

\section{Results}

\subsection{Descriptions}

SPSS 24 was used to analyse the data. As the analyses were exploratory, the study was not preregistered. Initial Kruskal-Wallis analyses were preformed to explore group differences between the autistic, SPD and TD groups across sensory reactivity and mental health. Results found no differences between the autistic and SPD groups, and as such these groups were combined and analysed as a neurodevelopmental sample. A further overview of the ASD, SPD and TD group comparisons can be found in Supplementary Tables 1 and 2.

A Shapiro-Wilks test indicated that the data were not normally distributed $(p<0.01)$, and as such nonparametric tests have been used for the analyses. Partial correlation analysis was used to understand the relationship between hyper-reactivity, hypo-reactivity, sensory seeking and mental health diagnoses. Please note that for the below analyses we make no adjustment for multiple comparisons as there remains ongoing debate as to whether and how these adjustments should be made, particularly as multiple comparison adjustments may lead to greater errors of interpretation when data are actual observations of nature [32-34].

Given the difference in IQ distribution, analyses were performed to examine the influence IQ had on the data. Correlational analyses were performed between Full Scale IQ scores and CSI-4 and SP 
raw scores. Moderate effects were detected in the NDC group for depression $(r=0.34,95 \% \mathrm{Cl}$ $[.068, .677], p=0.020)$, in the TD group for total sensory reactivity $(r=0.46,95 \% \mathrm{Cl}[.128, .711], p=$ $0.005)$ and in the TD group for inattention ( $r=-0.34,95 \% \mathrm{Cl}[-.604,-.006], \mathrm{p}=0.031)$. As such, for all following correlational analyses we have used the original combined data controlling for IQ.

\subsubsection{Sensory Profile}

In order to explore categorical differences indicative of diagnostic descriptions in sensory reactivity between NDC and typically developing (TD) groups, and to understand prevalence rates of sensory difficulties in our sample, Sensory Profile raw scores were analysed to reveal percentages of participants whose scores met cut-off for sensory reactivity as defined by Dunn [26] (Table 1). Cut-off scores for total sensory processing were derived as per construct cut-offs detailed in Dunn [26]. Probable difference scores lie between one standard deviation and two standard deviations below the mean of Dunn's sample and definite difference scores lie two standard deviations below the mean $(M=131.30, S D=8.73 ; 1 S D=122.57,2 S D=113.84)$. The mean score for the NDC group was 109.72 (SD = 14.93), and for the TD group was 130.86 (SD = 9.33). Percentages of participants meeting the threshold for probable or definite sensory reactivity difficulties across both construct and domain levels were consistently larger for the NDC group than for the TD group. The KruskalWallis test was used to explore these differences, and differences were detected across all sensory constructs and domains: sensory seeking $[\chi 2=18.75, p<0.001]$, hypo-reactivity $[\chi 2=33.08, p<$ $0.001]$, hyper-reactivity [ $\chi 2=13.14, p<0.001]$, total sensory reactivity $[\chi 2=41.14, p<0.001]$, auditory $[\chi 2=39.63, p<0.001]$, visual $[\chi 2=13.93, p<0.001]$, touch $[\chi 2=32.94, p<0.001]$ and oral $[x 2=24.24, p<0.001]$.

Table 1 Percentages of participants meeting probable or definite cut-off scores for sensory processing difficulties on the Sensory Profile with total comparison $p$ values of Kruskal-Wallis test.

\begin{tabular}{|c|c|c|c|c|c|c|c|c|}
\hline & NDC (\%) & & & TD (\%) & & & & \\
\hline & Probable & Definite & Total & Probable & Definite & Total & $x^{2}$ & $p$ \\
\hline Sensory seeking & 26.5 & 26.5 & 53.1 & 11.9 & 0.0 & 11.9 & 18.75 & $<.001$ \\
\hline Hypo-reactivity & 34.7 & 30.6 & 65.3 & 2.4 & 2.4 & 4.8 & 33.08 & $<.001$ \\
\hline Hyper-reactivity & 14.6 & 20.8 & 35.4 & 4.8 & 0.0 & 4.8 & 13.14 & $<.001$ \\
\hline $\begin{array}{ll}\text { Total sensory } \\
\text { reactivity }\end{array}$ & 25.0 & 58.3 & 83.3 & 11.9 & 4.8 & 16.7 & 41.14 & $<.001$ \\
\hline
\end{tabular}

Note. Cut-off scores for total sensory processing were derived as per construct cut-offs detailed in Dunn [26]. Probable difference scores lie between 1SD and 2SD below the mean of the TD group and definite difference scores lie 2 SD below the mean of the TD group $(M=131.30, S D=$ 8.73).

\subsubsection{CSI-4}

CSI-4 symptom count scores, derived from item CSI-4 endorsement, were analysed to explore categorical differences in percentages of participants meeting screening cut-off scores for mental health symptoms warranting further investigation, and to understand prevalence rates of mental 
health symptoms in our sample (Table 2). The Kruskal-Wallis test was used to explore these differences, and differences were detected for inattention [ $\chi 2=42.37, p<0.001$ ], hyperactivity [ $\chi 2$ $=39.81, p<0.001]$, ADHD [ $\chi 2=48.45, p<0.001]$, ODD $[\chi 2=9.82, p=0.002], C D[\chi 2=5.43, p=$ $0.020], \operatorname{GAD}[\chi 2=15.57, p<0.001]$, social phobia [ $\chi 2=9.72, p=0.002], \operatorname{OCD}[\chi 2=4.01, p=0.045]$, motor tic $[\chi 2=10.84, p=0.001]$, vocal tic $[\chi 2=5.91, p=0.015]$ and depression $[\chi 2=11.17, p=$ 0.001 ]. No differences were detected between groups in obsessions [ $\chi 2=2.84, p=0.092]$, compulsions [ $\chi 2=1.64, p=0.201]$, or PTSD [ $\chi 2=2.58, p=0.108]$.

Table 2 Percentages of participants meeting screening cut-off scores of mental health symptoms on the CSI warranting further investigation with comparison $p$ values of a Kruskal-Wallis test.

\begin{tabular}{lllll}
\hline & NDC (\%) & TD (\%) & $\boldsymbol{\chi}^{2}$ & $\boldsymbol{p}$ \\
\hline Inattention & 53.1 & 2.4 & 42.37 & $<.001$ \\
Hyperactivity & 33.3 & 2.4 & 39.81 & $<.001$ \\
ADHD & 37.5 & 2.4 & 48.45 & $<.001$ \\
ODD & 29.2 & 2.4 & 9.82 & .002 \\
CD & 12.5 & 2.4 & 5.43 & .020 \\
GAD & 40.4 & 7.1 & 15.57 & $<.001$ \\
Social Phobia & 20.4 & 2.4 & 9.72 & .002 \\
Obsessions & 29.2 & 14.3 & 2.84 & .092 \\
Compulsions & 12.5 & 4.9 & 1.64 & .201 \\
OCD & & & 4.01 & .045 \\
PTSD & 18.8 & 7.1 & 2.58 & .108 \\
Motor Tic & 22.9 & 2.4 & 10.84 & .001 \\
Vocal Tic & 22.9 & 4.9 & 5.91 & .015 \\
Depression & 14.6 & 2.4 & 11.17 & .001 \\
\hline
\end{tabular}

Note: $A D H D=$ Attention Deficit Hyperactivity Disorder; ODD = Oppositional Defiance Disorder; $\mathrm{CD}=$ Conduct Disorder; $\mathrm{GAD}=$ Generalised Anxiety Disorder; $\mathrm{OCD}=$ Obsessive Compulsive Disorder; PTSD = Posttraumatic Stress Disorder.

OCD is a composite of obsessions and compulsions; however, the CSI does not include cut-off scoring for OCD.

Depression is a composite of two sets of criteria outlined in the CSI manual [30].

\subsection{Sensory Reactivity and Mental Health}

In order to examine any divergence in the relationship between sensory reactivity and mental health symptoms across the NDC and typically developing groups, partial correlational analyses controlling for IQ were conducted between the Sensory Profile construct raw scores and the CSI-4 mental health construct symptom count scores (Table 3). It is worth noting that due to the reversed scoring of the SP, negative coefficients indicate that an increase in sensory reactivity relate to a decrease in mental health wellbeing. 


\subsubsection{Hyper-Reactivity and Mental Health}

A weak negative effect was detected for GAD in the NDC group ( $p r=-0.34,95 \% \mathrm{Cl}[-.851,-.015]$, $p=0.032)$, and a weak negative effect was detected in the TD group for ODD $(p r=-0.36,95 \% \mathrm{Cl}$ $[-.761,-.249], p=0.023)$.

\subsubsection{Hypo-Reactivity and Mental Health}

Weak to moderate negative effects were detected in the NDC group for ODD $(\mathrm{pr}=-0.36,95 \% \mathrm{Cl}$ $[-.581,-.035], \mathrm{p}=0.014)$, social phobia ( $\mathrm{pr}=-0.40,95 \% \mathrm{Cl}[-.633,-.136], \mathrm{p}=0.006)$, and depression ( $p r=-0.48,95 \% \mathrm{Cl}[-.673,-.202], p=0.001)$. Weak to moderate negative effects were detected in the TD group for vocal tic ( $p r=-0.60,95 \% \mathrm{Cl}[-.842,-.504], \mathrm{p}<0.001)$ and $\mathrm{GAD}(\mathrm{pr}=-0.33,95 \% \mathrm{Cl}$ $[-.747,-.035], p=0.035)$.

\subsubsection{Sensory Seeking and Mental Health}

Within the sensory seeking construct, moderate to large negative effects were detected in the NDC group for inattention ( $\mathrm{pr}=-0.44,95 \% \mathrm{Cl}[-.636,-.203], \mathrm{p}=0.002)$, hyperactivity $(\mathrm{pr}=-0.70,95 \%$ $\mathrm{Cl}[-.809,-.576], \mathrm{p}<0.001), \mathrm{ADHD}(\mathrm{pr}=-0.65,95 \% \mathrm{Cl}[-.784,-.497], \mathrm{p}<0.001), \mathrm{ODD}(\mathrm{pr}=-0.49,95 \%$ $\mathrm{Cl}[-.678,-.280], \mathrm{p}=0.001), \mathrm{CD}(\mathrm{pr}=-0.48,95 \% \mathrm{Cl}[-.689,-.194], \mathrm{p}=0.001), \mathrm{GAD}(\mathrm{pr}=-0.38,95 \% \mathrm{Cl}$ $[-.633,-.120], p=0.010)$, obsessions ( $p r=-0.36,95 \% \mathrm{Cl}[-.585,-.050], p=0.014)$, PTSD $(p r=-0.36$, $95 \% \mathrm{Cl}[-.595,-.067], \mathrm{p}=0.015)$, motor tic ( $\mathrm{pr}=-0.45,95 \% \mathrm{Cl}[-.632,-.216], \mathrm{p}=0.002)$, and depression ( $\mathrm{pr}=-0.47,95 \% \mathrm{Cl}[-.664,-.238], \mathrm{p}=0.001$ ). No effects were detected for sensory seeking in the TD group.

\subsubsection{Total Sensory Reactivity and Mental Health}

In the NDC group, weak to moderate negative effects were detected between total sensory reactivity and inattention ( $\mathrm{pr}=-0.41,95 \% \mathrm{Cl}[-.622,-.148], \mathrm{p}=0.005)$, hyperactivity $(\mathrm{pr}=-0.58,95 \%$ $\mathrm{Cl}[-.719,-.396], \mathrm{p}<0.001), \mathrm{ADHD}(\mathrm{pr}=-0.56,95 \% \mathrm{Cl}[-.726,-.352], \mathrm{p}<0.001)$, ODD $(\mathrm{pr}=-0.50,95 \%$ $\mathrm{Cl}[-.699,-.211], \mathrm{p}=0.001), \mathrm{CD}(\mathrm{pr}=-0.41,95 \% \mathrm{Cl}[-.644,-.107], \mathrm{p}=0.005), \mathrm{GAD}(\mathrm{pr}=-0.44,95 \% \mathrm{Cl}$ $[-.637,-.200], p=0.002)$, obsessions ( $p r=-0.39,95 \% \mathrm{Cl}[-.611,-.095], \mathrm{p}=0.009)$, PTSD $(\mathrm{pr}=-0.35$, $95 \% \mathrm{Cl}[-.629,-.017], \mathrm{p}=0.017)$, motor tic ( $\mathrm{pr}=-0.51,95 \% \mathrm{Cl}[-.704,-.249], \mathrm{p}<0.001)$ and depression ( $\mathrm{pr}=-0.52,95 \% \mathrm{Cl}[-.685,-.281], \mathrm{p}<0.001)$. No effects were detected for total sensory reactivity and mental health symptoms in the TD group.

Table 3 Partial correlations between parent-reported sensory processing and mental health constructs in NDC and TD groups, controlling for IQ, using the Sensory Profile and the Child Symptom Inventory.

\begin{tabular}{|c|c|c|c|c|c|c|c|c|}
\hline & \multicolumn{2}{|c|}{ Sensory Seeking } & \multicolumn{2}{|c|}{ Hypo-reactivity } & \multicolumn{2}{|c|}{ Hyper-reactivity } & \multirow{2}{*}{$\begin{array}{l}\begin{array}{l}\text { Total } \\
\text { reactivity }\end{array} \\
\text { NDC }\end{array}$} & \multirow{2}{*}{$\begin{array}{l}\text { sensory } \\
\underline{\text { TD }}\end{array}$} \\
\hline & $\underline{\text { NDC }}$ & $\underline{\text { TD }}$ & $\underline{\text { NDC }}$ & $\underline{\text { TD }}$ & NDC & $\underline{\text { TD }}$ & & \\
\hline Inattention & $-0.44 * *$ & 0.05 & -0.22 & 0.08 & $\overline{0.06}$ & 0.15 & $-0.41 * *$ & $\overline{0.09}$ \\
\hline
\end{tabular}




\begin{tabular}{lllllllll}
\hline Hyperactivity & $-0.70^{* * *}$ & -0.18 & -0.11 & -0.08 & 0.09 & 0.03 & $-0.58^{* * *}$ & -0.16 \\
ADHD & $-0.65^{* * *}$ & -0.08 & -0.19 & 0.01 & 0.08 & 0.13 & $-0.56^{* * *}$ & -0.04 \\
ODD & $-0.49 * *$ & -0.21 & $-0.36^{*}$ & -0.15 & 0.08 & $-0.36^{*}$ & $-0.50^{* *}$ & -0.27 \\
CD & $-0.48^{* *}$ & -0.02 & -0.16 & 0.07 & 0.13 & -0.13 & $-0.41^{* *}$ & -0.02 \\
GAD & $-0.38^{*}$ & 0.01 & -0.22 & $-0.33^{*}$ & $-0.34 *$ & -0.28 & $-0.44^{* *}$ & -0.14 \\
Social Phobia & -0.12 & -0.22 & $-0.40 * *$ & 0.07 & -0.29 & -0.02 & -0.29 & -0.16 \\
Obsessions & $-0.36^{*}$ & 0.03 & -0.11 & -0.14 & -0.26 & -0.27 & $-0.39 * *$ & -0.06 \\
Compulsions & -0.01 & 0.25 & 0.04 & 0.13 & 0.12 & -0.04 & 0.03 & 0.23 \\
OCD & -0.28 & 0.14 & -0.06 & -0.05 & -0.13 & -0.22 & -0.28 & 0.06 \\
PTSD & $-0.36 *$ & 0.06 & -0.16 & 0.05 & -0.06 & -0.06 & $-0.35 *$ & 0.06 \\
Motor Tic & $-0.45^{* *}$ & & -0.25 & & -0.28 & & $-0.51 * * *$ & \\
Vocal Tic & -0.25 & -0.05 & 0.04 & $-0.60 * * *$ & -0.23 & -0.25 & -0.24 & -0.24 \\
Depression & $-0.47^{* *}$ & 0.13 & $-0.48 * *$ & 0.12 & -0.07 & -0.15 & $-0.52 * * *$ & 0.11 \\
\hline
\end{tabular}

Note. No correlation was completed for the TD group in motor tics as no motor tic symptoms were identified in that group.

$*=p<.05 ; * *=p<.01 ; * * *=p<.001$

$A D H D=$ Attention Deficit Hyperactivity Disorder $; O D D=$ Oppositional Defiance Disorder $C D=$ Conduct Disorder; GAD = Generalised Anxiety Disorder; OCD = Obsessive Compulsive Disorder, PTSD = posttraumatic stress disorder.

\subsubsection{Shared Sensory Reactivity and Mental Health}

Where mental health symptoms were found to have a relationship with more than one sensory construct, multiple regression analyses were conducted to understand how each sensory construct adds to the prediction of mental health. A relationship between mental health symptoms and multiple sensory constructs was found for depression (sensory seeking and hypo-reactivity), GAD (sensory seeking and hyper-reactivity) and ODD (sensory seeking and hypo-reactivity).

A multiple linear regression was conducted with depression as the outcome variable, and sensory seeking and hypo-reactivity as predictor variables. Sensory seeking and hypo-reactivity explained a significant portion (19\%) of the variance seen in depressive scores $\left(R^{2}\right.$ adjusted $=.19, F(2,45)=6.49$, $p=.003)$. It was found that sensory seeking significantly predicted depression scores $(\beta=-.29, t(45)$ $=-2.06, p=.045)$, as did hypo-reactivity $(\beta=-.29, t(45)=-2.05, p=.046)$.

A multiple linear regression was conducted with GAD as the outcome variable, and sensory seeking and hyper-reactivity as predictor variables. Sensory seeking and hyper-reactivity explained a significant portion (16\%) of the variance seen in GAD scores $\left(R^{2}\right.$ adjusted $=.16, F(2,44)=4.19, p$ $=.022)$. It was found that sensory seeking significantly predicted GAD scores $(\beta=-.32, t(44)=-2.33$, $p=.024)$, however, hyper-reactivity did not significantly predict GAD scores $(\beta=-.23, t(44)=-1.67$, $p=.102)$.

A multiple linear regression was conducted with ODD as the outcome variable, and sensory seeking and hypo-reactivity as predictor variables. Sensory seeking and hypo-reactivity explained a 
significant portion (22\%) of the variance seen in ODD scores $\left(R^{2}\right.$ adjusted $=.22, F(2,45)=6.37, p$ $=.004)$. It was found that sensory seeking significantly predicted ODD scores $(\beta=-.38, t(45)=-2.69$, $p=.010)$, however, hypo-reactivity did not significantly predict ODD scores $(\beta=-.19, t(45)=-1.27$, $\mathrm{p}=.212)$.

\section{Discussion}

The objectives of this study were to explore the association between sensory reactivity and mental health symptoms across two paediatric cohorts: children with a neurodevelopmental condition and typically developing children. For the first time this study has revealed a relationship between mental health symptoms in paediatric cohorts outside of anxiety, and sensory seeking and hypo-reactivity across diagnoses. This underscores the intrinsic link between mental health conditions and broad sensory reactivity. It also provides a preliminary indication there are specific unique sensory-based profiles of mental health. Our results are novel in that they highlight that the relationship between sensory seeking and mental health symptoms may be much greater than previous research has suggested, and warrant further exploration.

\subsection{Sensory Reactivity}

Overall, 4 out of 5 NDC (83.3\%) children met the threshold for total sensory symptoms on the Sensory Profile, set at one standard deviation above the mean, compared to $16 \%$ of the TD group. Per inclusion criteria, SPD participants had to meet cut-off scores on one of the sensory subscales on the Sensory Profile. In line with previous studies identifying sensory hypo-reactivity at elevated levels in children with NDC, hypo-reactivity in the current study was identified as being the highest scored sensory construct for NDC participants (65.3\%) [35].

\subsection{Mental Health}

For mental health domains, participants with an NDC were more likely to meet screening criteria for a mental health condition than the TD group. Inattention was the highest rated symptom in the NDC group (53.1\%), whereas obsessions were the highest rated symptom in the TD group (14.3\%). GAD was the highest rated condition in the NDC group (40.4\%), and GAD and PTSD in the TD (7.1\%) group.

\subsection{Sensory Reactivity and Mental Health}

Our results echo the established understanding that NDC youth experience greater sensory and mental health difficulties than their typically developing peers. We found a relationship between sensory hyper-reactivity and mental health constructs. Specifically, sensory hyper-reactivity was correlated to GAD, similar to previous ASC and SPD research into sensory over-responsivity/hyperreactivity and constructs such as anxiety [19-21, 36, 37]. The hyper-reactivity/anxiety relationship has now been shown to be evident across age ranges, including in young children [21, 36], adolescents [38-40], adults [20], with this study showing this relationship in older children as well. Further, this relationship also appears independent of the measure of anxiety used, with previous research using a variety of anxiety measures including the Infant Toddler Social and Emotional Assessment (ITSEA), Toddler Behavior Assessment Questionnaire (TBAQ), Liebowitz Social Anxiety 
Scale (LSAS), State-Trait Anxiety Inventory (STAI), Revised Children's Manifest Anxiety Scale (RCMAS), Spence Children's Anxiety Scale (SCAS), or Diagnostic Interview Schedule for Children (DISC) [21, 36, 37].

Regarding sensory hypo-reactivity, we found a link to internalising symptoms, showing greater hypo-reactivity was related to greater mental health symptoms, such as depression, within the NDC group. Correlations have previously been found for typically developing children in hypo-reactivity and externalising behaviour, such as would be expected in ODD and conduct disorder [41]. In typically developing children with, or at risk of developing, conduct disorder, research has shown accelerated habituation to repeated sensations, indicating a decreased sensitivity to stimuli $[42,43]$. Previous research has also found links in hypo-reactivity and low mood in autistic children [38, 44], whereby autistic children who found it difficult to register activity-oriented sensory input in a meaningful way were more likely to present with a more negative mood [44]. Again, there appears to be a link between sensory hypo-reactivity and depression, highlighting the need to take sensory reactivity differences into account when supporting individuals with low mood.

Interestingly, sensory seeking, an often-neglected area of research, was the sensory subtype that showed most associations to mental health symptoms, both internalising as well as externalising. Specifically, sensory seeking was correlated with inattention, hyperactivity, ADHD, ODD, CD, GAD, Obsessions, PTSD, Motor Tic and Depression in the NDC group, such that the more seeking, the more mental health symptoms. Further, in mental health symptoms which showed a relationship with more than one sensory construct, it was sensory seeking which significantly predicted mental health. Specifically, sensory seeking significantly predicted depression, GAD and ODD scores, whereas hyper-reactivity did not significantly predict GAD and hypo-reactivity did not significantly predict ODD. Whilst causal inferences cannot be made from cross-sectional data, the nature of sensory seeking is such that seekers do not have their sensory needs met organically, and therefore must pursue such stimuli to meet those needs. Therefore, a potential argument can be made that from a construct level in children with an NDC who present with externalising behaviours, anxiety and depression, the disparity of having a sensory need but not being able to effectively satisfy that need may be associated with the development of mental health symptomology. On the other hand, seeking could also be used as a coping mechanism to manage mental health symptoms and might have beneficial effects as anecdotal reports suggest. Clinically, it is also worth exploring rates of sensory reactivity differences in children presenting with mental health symptoms.

\subsection{Limitations}

This study was unique in that it explored the association between sensory reactivity and mental health symptoms across children with an NDC and TD children. However, there are several limitations noted. This study utilised parent-report measures of sensory reactivity and mental health. It is possible that there exists a bias towards over- or under-responding to sensory or mental health domains, or misattribution of related behaviours in responses [45]. A number of participants had a comorbid diagnosis of ADHD. Whilst we followed an RDoC approach, looking at symptoms of sensory reactivity as well as mental health, rather than diagnostic labels, it is worth noting that there may be an effect on the rates of ADHD in the NDC group. However, as this study was interested in the relationship between sensory reactivity and mental health, and did not set out to estimate prevalence rates of one or the other, this should not affect findings significantly. Given this study 
utilised parent-report data only, it is worth taking into consideration how these profiles may change when including self-report or observational/interview data. It is also acknowledged that whilst the CSI provides an acceptable foundation for understanding mental health symptoms, it is considered highly appropriate that future research investigating mental health constructs in autism and broader NDCs consider measures of mental health which have been specifically designed, or at least validated, with this population.

Further, due to sample size and data limitations, we were not able to explore possible gender, socioeconomic or cognitive learning differences across and between groups. Nevertheless, demographic risk factors may influence the emergence or trajectory of sensory reactivity or mental health symptoms; however, we were unable to evaluate the role of these risk factors on our sensory and mental health variables.

\subsection{Future Directions}

Our findings have implications for interventions with young children with an NDC. Sensory reactivity appears to be related to the symptoms of mental health, and it may be beneficial to assess sensory difficulties as part of more rigorous mental health assessments within mental health services. From a research perspective, it is critical for future studies to explore the causal relationship between sensory reactivity and mental health, particularly with sensory seeking. A greater understanding of the causal direction would allow for the exploration of whether addressing sensory needs in children presenting with an NDC early may be a key component in preventative mental health interventions. Further, studies with larger sample sizes and both genders are required to test sensory domain subtypes (i.e. auditory/visual hypo-reactivity, hyper-reactivity and seeking) to fully understand the sensory-mental health relationship. Larger sample sizes would also give more weight to specific unique sensory-based profiles of mental health conditions.

\section{Conclusions}

The objectives of this study were to explore the association between sensory reactivity and mental health symptoms across children with an NDC and TD children. There appears to be a relationship between sensory reactivity and mental health symptoms, which has clinical implications. There is also preliminary evidence of specific unique sensory-based profiles of mental health.

\section{Acknowledgments}

The authors would like to thank the participants, their families, and the ASD and SPD communities. We would also like to thank the support of the Wallace Research Foundation, NIMH, MQ: Transforming Mental Health and Autistica.

\section{Additional Materials}

The following additional materials are uploaded at the page of this paper.

1. Table S1: Percentages of ASD, SPD and TD participants meeting probable or definite cut-off scores for sensory processing difficulties on the Sensory Profile. 
2. Table S2: Percentages of ASD, SPD and TD participants meeting screening cut-off scores of mental health symptoms on the $\mathrm{CBI}-4$ warranting further investigation.

\section{Author Contributions}

EM and TT conceived of the study, participated in its design. Testing and data collection were performed by EM, TT and MG. TR performed the data analysis, interpretation and drafted the paper. EM, TT and MG provided critical revisions. All authors approved the final version of the manuscript for submission.

\section{Funding}

This work was funded by grants from the Wallace Research Foundation (EM, grant number P0511051), NIMH (EM, grant number K23 83890-01), MQ and Autistica (TT, grant number MQF17/6).

\section{Competing Interests}

The authors have declared that no competing interests exist.

\section{References}

1. Tavassoli T, Bellesheim K, Siper PM, Wang AT, Halpern D, Gorenstein M, et al. Measuring sensory reactivity in autism spectrum disorder: Application and simplification of a clinicianadministered sensory observation scale. J Autism Dev Disord. 2016; 46: 287-293.

2. Leekam SR, Nieto C, Libby SJ, Wing L, Gould J. Describing the sensory abnormalities of children and adults with autism. J Autism Dev Disord. 2007; 37: 894-910.

3. Marco EJ, Hinkley LB, Hill SS, Nagarajan SS. Sensory processing in autism: A review of neurophysiologic findings. Pediatr Res. 2011; 69: 48-54.

4. Tomchek SD, Dunn W. Sensory processing in children with and without autism: A comparative study using the short sensory profile. Am J Occup Ther. 2007; 61: 190-200.

5. Miller $\amalg$, Anzalone ME, Lane SJ, Cermak SA, Osten ET. Concept evolution in sensory integration: A proposed nosology for diagnosis. Am J Occup Ther. 2007; 61: 135-140.

6. Ahn RR, Miller LJ, Milberger S, Mclntosh DN. Prevalence of parents' perceptions of sensory processing disorders among kindergarten children. Am J Occup Ther. 2004; 58: 287-293.

7. Ahn RR, Miller $\amalg$, Milberger S, McIntosh DN, Nielsen DM, Schoen SA, et al. Perspectives on sensory processing disorder: A call for translational research. Front Integr Neurosci. 2009; 3: 22.

8. Russell AJ, Murphy CM, Wilson E, Gillan N, Brown C, Robertson DM, et al. The mental health of individuals referred for assessment of autism spectrum disorder in adulthood: A clinic report. Autism. 2016; 20: 623-627.

9. Kreiser NL, White SW. ASD traits and co-occurring psychopathology: The moderating role of gender. J Autism Dev Disord. 2015; 45: 3932-3938.

10. Lai MC, Kassee C, Besney R, Bonato S, Hull L, Mandy W, et al. Prevalence of co-occurring mental health diagnoses in the autism population: A systematic review and meta-analysis. Lancet Psychiatry. 2019; 6: 819-829. 
11. Caamaño M, Boada L, Merchán-Naranjo J, Moreno C, Llorente C, Moreno D, et al. Psychopathology in children and adolescents with ASD without mental retardation. J Autism Dev Disord. 2013; 43: 2442-2449.

12. Simonoff E, Pickles A, Charman T, Chandler S, Loucas T, Baird G. Psychiatric disorders in children with autism spectrum disorders: Prevalence, comorbidity, and associated factors in a population-derived sample. J Am Acad Child Adolesc Psychiatry. 2008; 47: 921-929.

13. Gouze KR, Hopkins J, LeBailly SA, Lavigne JV. Re-examining the epidemiology of sensory regulation dysfunction and comorbid psychopathology. J Abnorm Child Psychol. 2009; 37: 10771087.

14. Carter AS, Ben-Sasson A, Briggs-Gowan MJ. Sensory over-responsivity, psychopathology, and family impairment in school-aged children. J Am Acad Child Adolesc Psychiatry. 2011; 50: 12101219.

15. Cooper K, Smith LG, Russell A. Social identity, self-esteem, and mental health in autism. Eur J Soc Psychol. 2017; 47: 844-854.

16. Juranek J, Filipek PA, Berenji GR, Modahl C, Osann K, Spence MA. Association between amygdala volume and anxiety level: Magnetic resonance imaging (MRI) study in autistic children. J Child Neurol. 2006; 21: 1051-1058.

17. Yin J, Schaaf CP. Autism genetics - an overview. Prenat Diagn. 2017; 37: 14-30.

18. Gray DE. "Everybody just freezes. Everybody is just embarrassed": Felt and enacted stigma among parents of children with high functioning autism. Sociol Heal Illn. 2002; 24: 734-749.

19. Green SA, Ben-Sasson A. Anxiety disorders and sensory over-responsivity in children with autism spectrum disorders: Is there a causal relationship? J Autism Dev Disord. 2010; 40: 14951504.

20. Liss M, Timmel L, Baxley K, Killingsworth P. Sensory processing sensitivity and its relation to parental bonding, anxiety, and depression. Pers Individ Differ. 2005; 39: 1429-1439.

21. Goldsmith HH, Van Hulle CA, Arneson CL, Schreiber JE, Gernsbacher MA. A population-based twin study of parentally reported tactile and auditory defensiveness in young children. J Abnorm Child Psychol. 2006; 34: 378-392.

22. Harrison LA, Kats A, Williams ME, Aziz-Zadeh L. The importance of sensory processing in mental health: A proposed addition to the research domain criteria (RDoC) and suggestions for RDoC 2.0. Front Psychol. 2019; 10: 103.

23. Kenny L, Hattersley C, Molins B, Buckley C, Povey C, Pellicano E. Which terms should be used to describe autism? Perspectives from the UK autism community. Autism. 2016; 20: 442-462.

24. Rutter M, Bailey A, Lord $C$. The social communication questionnaire manual. Los Angeles, CA.: Western Psychological Services; 2003.

25. Lord C, Rutter M, DiLavore PC, Risi S, Gotham K, Bishop S, et al. Autism diagnostic observation schedule, second edition: ADOS-2. Torrance, CA: Western Psychological Services; 2012.

26. Wechsler D. Wechsler intelligence scale for children-fourth edition: WISC-IV. San Antonio, TX: The Psychological Corporation; 2003.

27. Dunn W. Sensory profile: User's manual. San Antonio, TX: Psychological Corporation; 1999.

28. Ben-Sasson A, Hen L, Fluss R, Cermak SA, Engel-Yeger B, Gal E. A meta-analysis of sensory modulation symptoms in individuals with autism spectrum disorders. J Autism Dev Disord. 2009; 39: 1-11. 
29. Ohl A, Butler C, Carney C, Jarmel E, Palmieri M, Pottheiser D, et al. Test-retest reliability of the sensory profile caregiver questionnaire. Am J Occup Ther. 2012; 66: 483-487.

30. Gadow KD, Sprafkin JN. Child symptom inventory 4: Screening and norms manual. Stony Brook, NY: Checkmate Plus; 2002.

31. Gadow KD. The Symptom Inventories: An annotated bibliography. Stony Brook, NY: Checkmate Plus; 2019.

32. Feise RJ. Do multiple outcome measures require $p$-value adjustment? BMC Med Res Methodol. 2002; 2: 1-4.

33. Rubin $M$. Do $\mathrm{p}$ values lose their meaning in exploratory analyses? It depends how you define the familywise error rate. Rev Gen Psychol. 2017; 21: 269-275.

34. Rothman KJ. No adjustments are needed for multiple comparisons. Epidemiology. 1990; 1: 4346.

35. Schoen SA, Miller $\sqcup$, Brett-Green B, Nielsen DM. Physiological and hehavioral differences in sensory integration processing: A comparison of children with autsm spectrum disorder and sensory modulation disorder. Front Integr Neurosci. 2009; 3: 29.

36. Green SA, Ben-Sasson A, Soto TW, Carter AS. Anxiety and sensory over-responsivity in toddlers with autism spectrum disorders: Bidirectional effects across time. J Autism Dev Disord. 2012; 42: 1112-1119.

37. Van Hulle CA, Schmidt NL, Goldsmith HH. Is sensory over-responsivity distinguishable from childhood behavior problems? A phenotypic and genetic analysis. J Child Psychol Psychiatry Allied Discip. 2012; 53: 64-72.

38. Pfeiffer B, Kinnealey M, Reed C, Herzberg G. Sensory modulation and affective disorders in children and adolescents with Asperger's disorder. Am J Occup Ther. 2005; 59: 335-345.

39. Pickard H, Hirsch C, Simonoff E, Happé F. Exploring the cognitive, emotional and sensory correlates of social anxiety in autistic and neurotypical adolescents. J Child Psychol Psychiatry Allied Discip. 2020; 61:1317-1327.

40. MacLennan K, Roach $L$, Tavassoli $T$. The relationship between sensory reactivity differences and anxiety subtypes in autistic children. Autism Res. 2020; 13: 785-795.

41. Fox C, Snow PC, Holland K. The relationship between sensory processing difficulties and behaviour in children aged 5-9 who are at risk of developing conduct disorder. Emot Behav Diffic. 2014; 19: 71-88.

42. Zahn TP, Kruesi MJ. Autonomic activity in boys with disruptive behavior disorders. Psychophysiology. 1993; 30: 605-614.

43. Herpertz SC, Mueller B, Wenning B, Qunaibi M, Lichterfeld C, Herpertz-Dahlmann B. Autonomic responses in boys with externalizing disorders. J Neural Transm. 2003; 110: 1181-1195.

44. Chuang IC, Tseng MH, Lu L, Shieh JY. Sensory correlates of difficult temperament characteristics in preschool children with autism. Res Autism Spectr Disord. 2012; 6: 988-995.

45. Stokes J, Pogge D, Wecksell B, Zaccario M. Parent-child discrepancies in report of psychopathology: The contributions of response bias and parenting stress. J Pers Assess. 2011; 93: 527-536. 


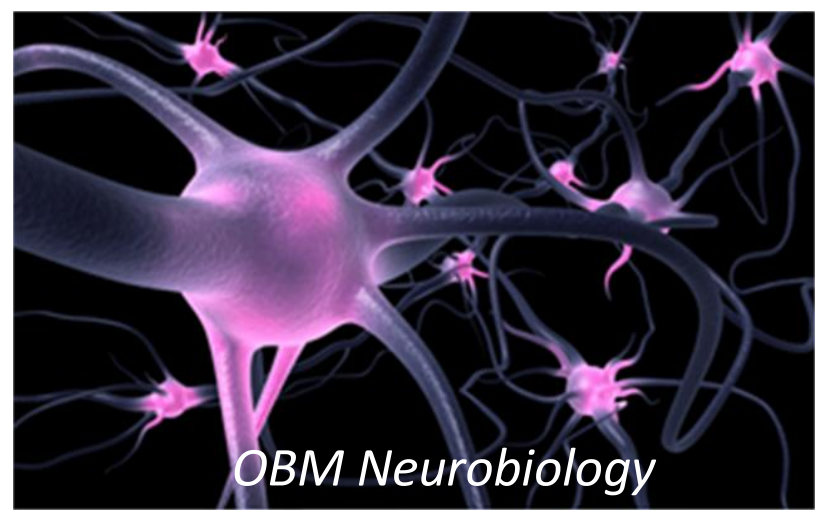

Enjoy OBM Neurobiology by:

1. Submitting a manuscript

2. Joining volunteer reviewer bank

3. Joining Editorial Board

4. Guest editing a special issue

For more details, please visit:

http://www.lidsen.com/journals/neurobiology 